\title{
THE DEMOGRAPHIC COMPONENTS OF POPULATION AGING IN CHINA
}

\begin{abstract}
Past trends in ferrility and mortality in China have led to an age composition that will age rapidly in the coming decades. In this paper we examine measures of population aging in China from 1953 to 1982 , and then project population aging to the year 2050 using a cohort-components methodology. The projected measures of population aging that result from these forecasts are then decomposed into the relative contributions that are made to these changes by past, present, and future trends in fertility and mortality. Results indicate that China's population will age at an unprecedented rate over the next 70 years, both in terms of the absolute size of the elderly population and their proportion of the total population. At least 50 percent of the projected increase in population aging in China between 1980 and 2050 will be a product of the momentum for aging that is already built into the present age structure and vital rates. However, prospective trends in the measures of population aging become increasingly more sensitive to varying assumptions about fertility and mortality with time, and as older age groups are considered. This analysis provides the demographic basis for evaluating the possible effects of population aging on health care, social security, and other social and economic issues.
\end{abstract}

\section{INTRODUCTION}

After the cultural revolution in the late 1970s Chinese policy-makers and scientists expressed a renewed concem over the size and growth rate of the nation's population. At that time Chinese scientists recognized that populationrelated problems needed to be addressed with urgency, and in response the Chinese government quickly set into motion policies that would produce rapid declines in growth rates and eventually lead the nation to an "optimum" population size (Ming 1979; Chen 1979a). ${ }^{1}$ With the understanding that the momentum for population growth would be extremely difficult to manage, and given limited resources and a general lack of concern and knowledge about other possible demographic consequences of their actions, Chinese officials decided to focus their attention exclusively on reducing the population's birth rate. The result was China's now well-known one-child family policy.

China's one-child policy was implemented in 1979 in an effort to meet the target of an optimum population of 1.2 billion set for the year 2000 (Chen 1979a; Guanming Daily 1979). In addition to this optimum population size, the government expected to achieve zero population growth by the year 2000 and a targeted growth rate for the $1980 \mathrm{~s}$ ranging from $0.5 \%$ to $1.0 \%$ depending on who was setting the targets. ${ }^{2}$ These targets were later supported by population forecasts made by Chinese scientists which illustrated how varying trends in fertility would lead to a wide range of optimum population levels, and that a target total fertility rate (TFR) of 1.5 would eventually lead to a stabilized

Journal of Cross-Cultural Gerontology 4: 307-334, 1989.

(C) 1989 Kluwer Academic Publishers. Printed in the Netherlands. 
population of 1.2 billion people (Song et al. 1980). In effect, these forecasts represented the ex post facto justification for the strict one-child policy that was introduced two years earlier.

While the one-child policy was advertised as a series of positive sanctions that would 'encourage' couples to delay marriage and childbearing and have only one child, ${ }^{3}$ it has been noted by Banister (1986) and revealed in the Chinese literature (NFRB 1983; KMJP 1979), that more extreme family planning measures have been used in some provinces (e.g. required sterilization of either the husband or the wife for couples who already have two children, the required insertion of IUDs in women with one child, and abortions for women who became pregnant without official permission). The implementation of the onechild policy illustrates the tenacity with which the Chinese government is capable of altering the nation's demographic processes. In any event, primarily as a result of this and other family planning policies, the total fertility rate (TFR) declined from a level of 6.0 in 1960 to about 2.1 today (Bongaarts and Greenhalgh 1985). Numerous articles have appeared in the Chinese and western demographic literature which have evaluated both the effectiveness of this policy in reducing the rate of population growth, and the social and economic implications of subsequent perturbations in China's age composition (Aird 1986; Banister 1987, 1988; Bongaarts and Greenhalgh 1985; Coale 1981; Keyfitz 1984; Liang et al. 1986; Wei 1987; Wu 1986; Wu and Xu 1987).

When the one-child policy was first introduced, several Chinese scientists noted that if such a program were successful it would lead to a rapid narrowing of the base of the age structure, and an acceleration of population aging to the point where an estimated $20-30 \%$ of China's total population would be aged 60 and over within 50 years of the implementation of the policy (Hu et al. 1980). In spite of these early warnings, both government officials and some scientists (who were actually responsible for making these forecasts) insisted at the time that either there was no need to worry about the problem until after the year 2020 (Chen 1979b; FBIS 1980), ${ }^{4}$ or that population aging could be avoided by increasing fertility in the future (Hu et al. 1980; Tian 1980). Some Chinese scientists have even argued more recently that the size of the elderly population will remain constant for the next 45 years (China Population Newsletter 1986), or that the best insurance against population aging in the distant future is a successful family planning program today (Hao and Wang 1987; RKYJ 1987). Others have noted that because the percentage of the population aged 65 and over in China will not exceed the international definition of an aged population (e.g. where $7 \%$ of the total population is aged 65 and over) until early into the next century, then population aging need not be considered a problem at this time (China Population Newsletter 1986; FBIS 1980). Even today there are still some Chinese scientists and political officials who ignore the basic demographic principles associated with shifts in age composition, and fail to recognize the policy implications of the impending explosive growth of the absolute size of their elderly population. 
In spite of these efforts to dismiss the issue of population aging, within 5 years of the implementation of the one-child policy several well-known Chinese demographers presented forecasts which illustrated that if low fertility rates persisted past the middle of the next century, the population aged 65 and over would inevitably comprise from $36-41$ percent of the total population sometime between 2045 and 2080 (Gui 1983). Other well-known Chinese demographers and political officials quickly echoed these concerns about population aging and recognized that the problem was serious enough to warrant either the development of new policies to deal with population aging, or a change in the one-child policy (Wu 1985; Wu 1984a; Wu 1984b; Tao 1984; Qie 1984; Tian 1984). New population projections then illustrated how the government could achieve an optimum population of under one billion people and at the same time slow the rate of population aging (Tian 1984). By eventually relaxing the stringent goals of the one-child policy fertility could gradually rise back to replacement levels, and population aging would begin to decelerate..$^{5}$ In short, there is now a major research effort underway in China to understand the social and economic problems associated with population aging, and to develop policies that will reduce its major impacts.

\section{POPULATION AGING IN CHINA: MAGNITUDE, MOMENTUM, MORBIDITY}

There are several reasons that the problems associated with population aging in China require prompt attention. The most apparent issue is the sheer magnitude of the problem or the absolute number of people involved. Even though China has a comparatively small percentage $(5.5 \%)$ of its total population currently over the age of 65 , this translates into about 59.8 million people, most of whom live in rural areas (Kinsella 1988). By comparison, in the United States there were 29.2 million persons aged 65 and over in 1986 (U.S. Bureau of the Census 1988, 13). Regardless of how fertility rates change during the next six decades, it has been estimated that the population aged 65 and over in China would still increase in absolute numbers to between 267.8 to 340.9 million persons by the year 2050, which would be about 20-22 percent of the total population (Banister 1987, 1988; Wu and Xu 1987). Consequently by the year 2050, the number of people aged 65 and older in China will certainly exceed the total number of people of all ages in the United States today. Since the number of people who will be over the age of 65 between 1985 and the year 2050 have already been born, these differences in projections of the size of the elderly population occur solely as a result of different assumptions about mortality. ${ }^{6}$

Figure 1 compares the observed size of the 65 and older population in China and the United States during selected time periods in the past, and projections of the elderly population for these countries made by different authors. While China's total population size was 3.65 times greater than that of the U.S. in 1953 , the population aged 65 and over was only 1.88 times the size of the 


\section{SUO!|!!W}
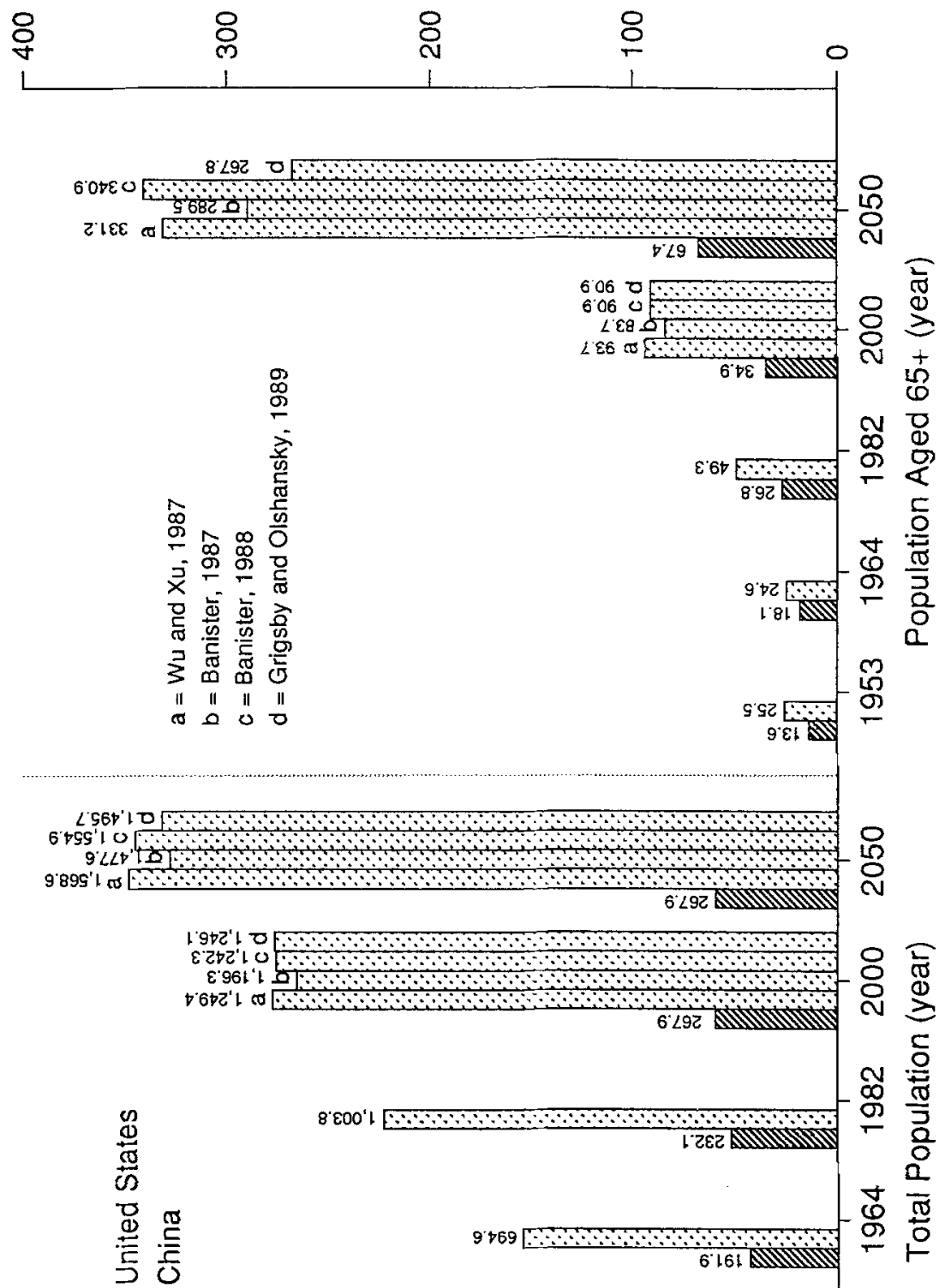

$$
\varepsilon 961 \text { เ }
$$

$6 \angle 9 Z$

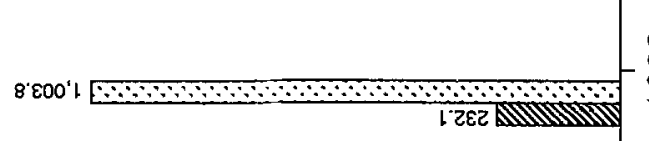

๙ $\frac{0}{0}$

\& $\overline{0}$

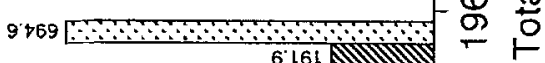

חू

9289

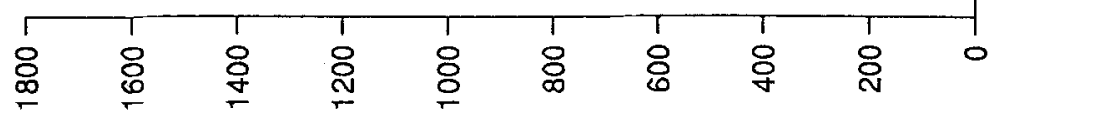


elderly population in the U.S. because fertility and mortality rates prior to 1953 produced a much younger age composition for China. Even though the elderly population of the U.S. is projected to exceed 67 million persons by the year 2050 , in China the size of the elderly population is projected to be more than four times greater, with at least 267 million persons over age 65 . The projected range of the size of the 65 and older population of China for the year 2050 is 267.8 million (Grigsby and Olshansky in this paper) to 340.9 million by Bannister (1988) - virtually all of which is attributable to variation in assumptions about mortality. ${ }^{7}$

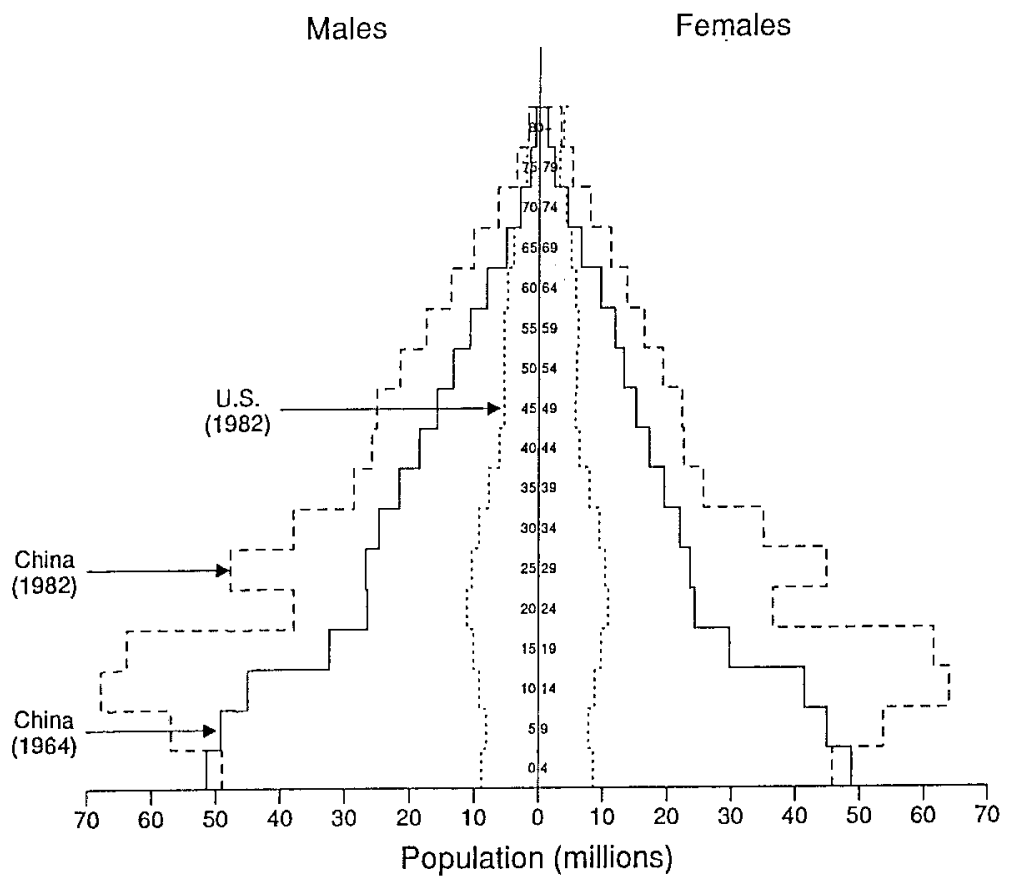

Figure 2.

In spite of the fact that the size of the elderly population of China nearly doubled from 1964 to 1982, the overall shape of the age compositions for the two time periods were remarkably similar (see Figure 2). The growth rate of the population in most age groups remained fairly constant during these years, thereby producing an expansion of the age pyramid while leaving constant the age-sex distribution of the population. However, from 1964 to 1982 the oldest age groups did experience a small proportional increase while the percentage distribution of the two youngest age groups did decline substantially. The population distribution of the U.S. in 1982 is presented here to illustrate differences in magnitude of the size of the various age groups. 


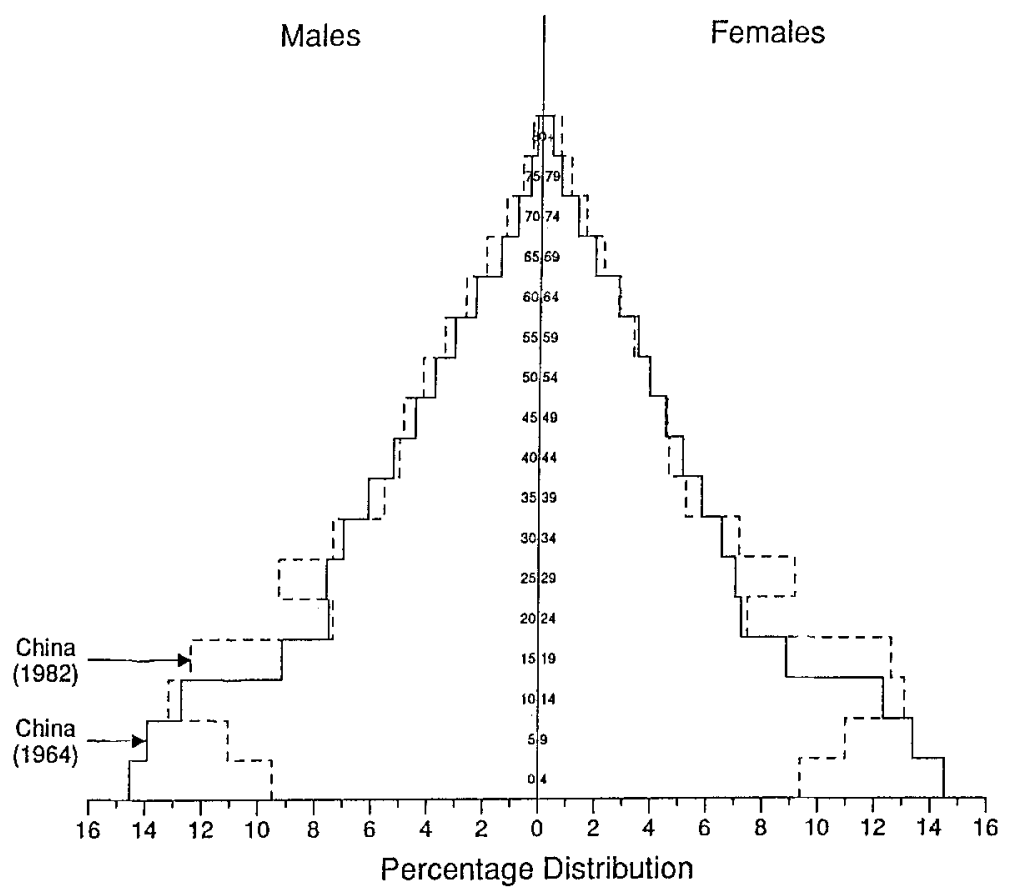

Figure 3.

Figure 3 illustrates this similarity in age compositions by comparing the percentage distribution of China's population by age and sex during these same years. Figures 2 and 3 illustrate that it is possible to experience population aging in terms of increases in the absolute size of the elderly population during a time in which the overall age distribution of the population does not change. If population aging were measured using only the percentage of the total population aged 65 and older, then one would conclude that China's population had not aged significantly from 1964 to 1982 . This was indeed the conclusion that was drawn by some Chinese policymakers and scientists (China Population Newsletter 1986; FBIS 1980). ${ }^{8}$ It is for this reason that more attention should be given to evaluating several different kinds of measures of population aging rather than focusing exclusively on the percentage of the total population that is elderly.

A second issue is the momentum for population aging that is already built into China's age structure, and the presence of a number of social, economic, and demographic factors that are likely to accelerate population aging beyond current projections. Past levels of fertility and mortality have led to an age composition today which is primed for rapid increases in all of the measures of population aging and an unprecedented increase in the absolute numbers of people over the age of 65 (See Figure 1). This aging process is inevitable as the extremely large birth cohorts of the period 1960 to 1970 begin to pass through 
the middle and older ages during the next 70 years. Population aging in China will also be accelerated by the rapid narrowing of the base of the age structure that is caused by the successful one-child family policy. This will bring rapid increases in measures of population aging which quantify shifts in age composition, as the narrowing of the base of the age pyramid will cause an increase in the relative proportion of the total population that is old. Finally, if in the next 60 years mortality rates begin to decline even marginally beyond current expectations for cohorts surviving to older age groups, then this will place a third and rather substantial upward pressure on the momentum for population aging in China. Under these conditions, both the absolute number of elderly persons and their percentage of the total population would increase as the apex of the age pyramid would be expanded.

In addition to problems of magnitude and momentum for population aging, China also faces the difficult task of financially supporting and meeting the acute and chronic morbidity requirements of a rapidly expanding elderly population, particularly with respect to the provision of long-term care. While life expectancy at birth in China resembles that of a developed country (70 years), the quality and delivery of health care are still more like that of a developing country. For example, while the total number of hospitals and health care workers increased since 1970, the majority of this increase took place in urban areas where less than one-fourth of the population live (Banister 1986). Many of the barefoot doctors who used to provide minimal health care in rural areas, have abandoned their medical work in favor of more financially rewarding jobs in agriculture (Banister 1986, 13). There is no health insurance coverage for those in rural areas who are not members of a cooperative medical plan, and the cooperative medical system that is in place has deteriorated in recent years (Banister 1986, 16). Finally, for years the Chinese government has been absorbing an increasing share of the money spent on health care, and this is expected to continue well into the future.

The rapid aging of China's population will add considerably to the strain on the nation's health care system. Either more funds will need to be provided by the government to handle the health care requirements of the elderly, or existing funds used for other purposes such as family planning, will need to be redirected toward health care for the aged. Additionally, acute and long-term health care facilities like those in developed countries that are designed to handle the unique and costly health care requirements of the elderly, are practically non-existent in China. In the absence of these kinds of facilities, and given the magnitude of the growth of the elderly population that is expected by the middle of the next century, it is anticipated that China will have a difficult time maintaining their relatively high health standards (Hsiao 1988).

Finally, it should be emphasized that China relies heavily on the family as an institution for providing both financial and social support for the elderly. A successful one-child policy will inevitably bring an increase in the number of childless elderly couples who will need state-funded financial support. ${ }^{9}$ It will 
also create an unfair burden on the children born during this era because many will face the unenviable responsibility of caring for four elderly parents, 1-2 of their own children, and any grandparents that may survive to much older ages (Wu and $\mathrm{Xu}$ 1987). Additionally, the pension system in China meets the requirements of only a relatively small portion of the nation's elderly (Banister $1988,5-6)$. There is increasing concern that the economy will not be able to support either the increases in expenditures for the growing population who will receive benefits under the existing pension scheme (e.g., social security, medical care, housing, transportation, etc.), nor is it likely that such a pension scheme could be expanded to provide even basic expenses for the remainder of the elderly population (Banister 1988; Wu 1984a; Zhao and Jiang 1986).

\section{MEASURING POPULATION AGING IN CHINA: 1953-1982}

The process of population aging refers generally to a shift in age composition in the direction of older ages. It typically occurs among nations that have experienced rapid population growth during the transition from high to low levels of fertility and mortality, and it is accelerated by declining mortality for older age groups. While there is no single demographic measure that qualifies as the best measure of population aging, certainly it has been common to examine trends in the relative proportions of the population that are defined as elderly. It is also reasonable to consider a number of other measures of populaton aging which provide additional insights into the various ways in which a population ages. In this section we follow trends in several measures of population aging in China from 1953 to 1982 , and discuss briefly the relative merits of these measures as they apply specifically to China.

One of the most common measures of population aging is the proportion of the total population that is old. The age which delimits "old" is often culturebound, although it often refers to the age of eligibility for a social or economic benefit, or an age when a large segment of the populaton has a significant physical, economic, or social need. The age of 65 is used most often by nations of both the developed and developing world to define the "old" or elderly population. In China, however, the age of 60 has been used to define the elderly population as urban employees tend to retire at that age (Wu and $\mathrm{Xu}$ 1987). More recent publications regarding population aging in China have tended to use the age of 65 as the lower bound of the older population (e.g. Song et al., 1985; Banister 1987).

The data in Table I illustrate that regardless of which age is used to define old, it is clear that the relative proportions of all of the age groups over the age of 55 in China decreased from 1953 to 1964, and then increased from 1964 to 1982 to signify that population aging had begun. The age of 55 was included in this table because this is the age at retirement for women working in some urban occupations. This trend toward a younger population followed by an aging population occurred primarily because of high birth rates and rapidly declining infant and 
child mortality rates in the $1950 \mathrm{~s}$, both of which caused an expansion at the base of the age pyramid. Lower birth rates beginning in the early 1970s began to narrow the base of the age structure and increase the relative proportion of the population that was old.

TABLE I

Measures of Population Aging in China

$(1953,1964,1982)$

\begin{tabular}{lrrr}
\hline $\begin{array}{l}\text { Measures of } \\
\text { Population Aging }\end{array}$ & $\begin{array}{c}1953 \\
(1)\end{array}$ & $\begin{array}{c}1964 \\
(2)\end{array}$ & $\begin{array}{c}1982 \\
(3)\end{array}$ \\
\hline Percent of the & & & \\
Population Aged & & & \\
$\quad 55+$ & 10.88 & 9.40 & 11.02 \\
$60+$ & 7.26 & 6.13 & 7.64 \\
$65+$ & 4.38 & 3.56 & 4.91 \\
$70+$ & 2.32 & 1.88 & 2.79 \\
$75+$ & 0.95 & 0.81 & 1.36 \\
$80+$ & 0.32 & 0.26 & 0.50 \\
P(75+)/P(65+) & 21.75 & 22.66 & 27.72 \\
P(80+)/P(65+) & 7.40 & 7.38 & 10.25 \\
$\quad 0-14$ & 35.92 & 40.69 & 33.60 \\
15-64 & 59.71 & 55.74 & 61.49 \\
P(65+)/P(0-14) & 12.19 & 8.76 & 14.61 \\
Median Age & 23.02 & 20.20 & 22.65 \\
Dependency Ratio & 67.49 & 79.40 & 62.62 \\
Index of Relative & & & 8.90 \\
$\quad$ Difference & - & 6.21 & 9.61 \\
Index of Dissimilarity & - & 5.86 & \\
\hline
\end{tabular}

Source: These measures were calculated from data published in Banister (1987, pp. 25, 27, 34).

Another measure of population aging is the relative age distribution of the elderly population itself. The measures used most often to quantify the aging of the aged is to take the ratio of the number of people aged $75+, 80+$, or $85+$ by the population aged 65 and over. Increases in these measures indicate that the relative distribution of the elderly population itself is aging. In China it is apparent that while the elderly population did not age much from 1953 to 1964 , by 1982 the "old-old" comprised almost 28 percent of the older population itself, an increase of over 5 percent from 1964. The proportion of the older population over age 80 also increased during this time period.

The proportion of persons in younger age groups, for example under age 15 or between ages 15 and 64 , also measure population age structure even though they do not take the elderly into account directly. Often the reason for studying the numbers or proportions of persons in these age groups is to answer policy questions, such as planning for school enrollments or for estimating the size of 
the labor force. Between 1953 and 1964, the proportion of the population under age 14 increased from 36 percent to 41 percent, but declined to 34 percent by 1982. The "working age" population aged 15-64 comprised approximately 60 percent of the total population in both 1953 and 1982, and was somewhat lower in 1964.

Several other kinds of ratio measures have also been constructed to measure population age structure. The most familiar to demographers is the standard dependency ratio, with the young (ages 0-14) and old (ages 65 and older) populations in the numerator and the "working age" (15-64) population in the denominator. The numerator is supposed to approximate the economically dependent population while the denominator represents the economically active population. However, since age is not a perfect indicator of economic activity, the dependency ratio does not measure dependency very accurately and it tends to perpetuate the negative stereotype of persons over age 65 as being dependent on persons aged 15 to 64 . Furthermore, the dependency ratio does not provide an accurate picture of the population age structure because the numerator contains both the young and old populations. Typically, increases in the proportion of older persons occur along with decreases in the proportion of young persons. These two effects tend to balance each other so that populations with very different age structures may have similar dependency ratios. Nevertheless, for policy purposes it may be useful to examine the dependency ratio, not necessarily as an indicator of population aging, but rather to see how it is affected by population aging. Between 1953 and 1964, the dependency ratio increased due to the rise in the proportion of younger persons and a decline in the proportion of working-age persons. By 1982 the dependency ratio had decreased even below its former level in 1953, as the proportion of younger persons declined while the working-age population increased in proportion.

Shryock and Siegel $(1980$, p. 234) suggested that the aged-to-youth ratio may be the best measure of population aging because this measure includes the two population subgroups which change the most during the demographic transition (e.g. the population aged $0-15$ and $65+$ ). Indeed, the aged-to-youth ratio declined in China between 1953 and 1964, as the population grew slightly younger, and it rose again between 1964 and 1982.

The average age of the population (either the median age or mean age) has intuitive appeal for summarizing the age structure. While the average age rarely, if ever, guides public policy (for instance, in determining an age of eligibility for entitlement programs), it is convenient for comparing the age structures of two or more populations. The average age also allows the researcher to measure population aging without having to define what age is old. The median age of the Chinese population decreased from 23 years in 1953 to 20 years in 1964, then rose to 23 years again by 1982 .

In addition to measuring population aging per se, it is also possible to measure differences in the age structure of a population across time and summarize them in a single index. The index of dissimilarity measures the proportion of a 
population in one time period that would have to "move" into other age groups in order to resemble the age structure of the same population during another time period. The index of relative difference summarizes the percentage difference within each age group across time. Both indexes depend on the number of age groups as well as the differences in the age structure. When comparing more than two populations, therefore, it is useful to have a single reference population (Shryock and Siegel 1980, pp. 232-233). The reference or standard population used in this case was the 1953 population of China. Since both of these indexes increased from 1964 to 1982 , this indicates that the change in the age structure of the Chinese population was greater between 1964 and 1982 then it was between 1953 and 1964.

In summary, virtually all of the measures of population aging presented in Table I demonstrate that the population of China grew younger between 1953 and 1964, and aged from 1964 to 1982. The data in Figure 3 indicate that the absolute size of the elderly population in China also increased substantially during this latter time period - further substantiating that the population aged. It should be noted that at least in China the changes in age composition that took place during this time of population aging actually led to a decrease in the dependency ratio - another example of how the dependency ratio does not always reflect population aging. In the next section we will examine the effects on measures of population aging, of a range of projection assumptions regarding the components of population change in China.

\section{PROJECTING POPULATION AGING IN CHINA: 1980-2050}

Population projections that have been made for China by various sources have often been made by using two or three fertility assumptions and only one mortality assumption to illustrate a range of outcomes (Banister 1986; 1987; Bongaarts and Greenhalgh 1985; Wu and Xu 1987). In China, just as in most other nations, future trends in mortality are just as uncertain as those of fertility. In fact, China already has a mortality schedule and a life expectancy at birth (of 70 years) that compares to that of many developed nations. The transition to this favorable mortality schedule occurred over a very short time period from 1949 to 1975 for reasons that are not entirely clear (Banister and Preston, 1981), and there is still the presence of relatively high mortality rates for younger age groups - indicating that there is considerable room for additional improvements in mortality. While Banister (1986) has argued that further improvements in mortality may occur more slowly in the future because of inadequate health care in rural areas and increased cigarette smoking, it is still possible that significant mortality declines may occur in the near future. Moreover, it has already been demonstrated that future trends in population aging in China will be very sensitive to minor variations in assumptions about mortality (Xu 1985). It is for these reasons that we have included high, medium, and low estimates of future trends in mortality in our population projections. 
Future trends in fertility are also subject to speculation. In this study we used the low, medium, and high assumptions about fertility in China that were made by the United Nations (1986) from 1980 to the year 2025, and then projected that each series would increase to a total fertility rate of 2.11 children per woman by 2050 . Migration was assumed to be zero. ${ }^{10}$ Details of both the fertility and mortality assumptions used in this study are provided in Table A-I in the appendix.

The combination of low fertility and low mortality is known to produce the greatest amount of population aging, while the high series of fertility and mortality will produce the least amount of population aging. All other combinations of fertility and mortality schedules result in age structures between these two extremes. The medium fertility and medium mortality assumptions were used to project a population age structure that is intermediate to these. The 1980 population of China (United Nations 1982) was projected forward in time using a cohort-components methodology (The Futures Group 1987). The projections presented here are similar to those of Aird (1986), Banister (1987) and Wu and Xu (1987) in terms of both the total projected population size and the projected shape of the age structures, taking into account the minor variation in assumptions.

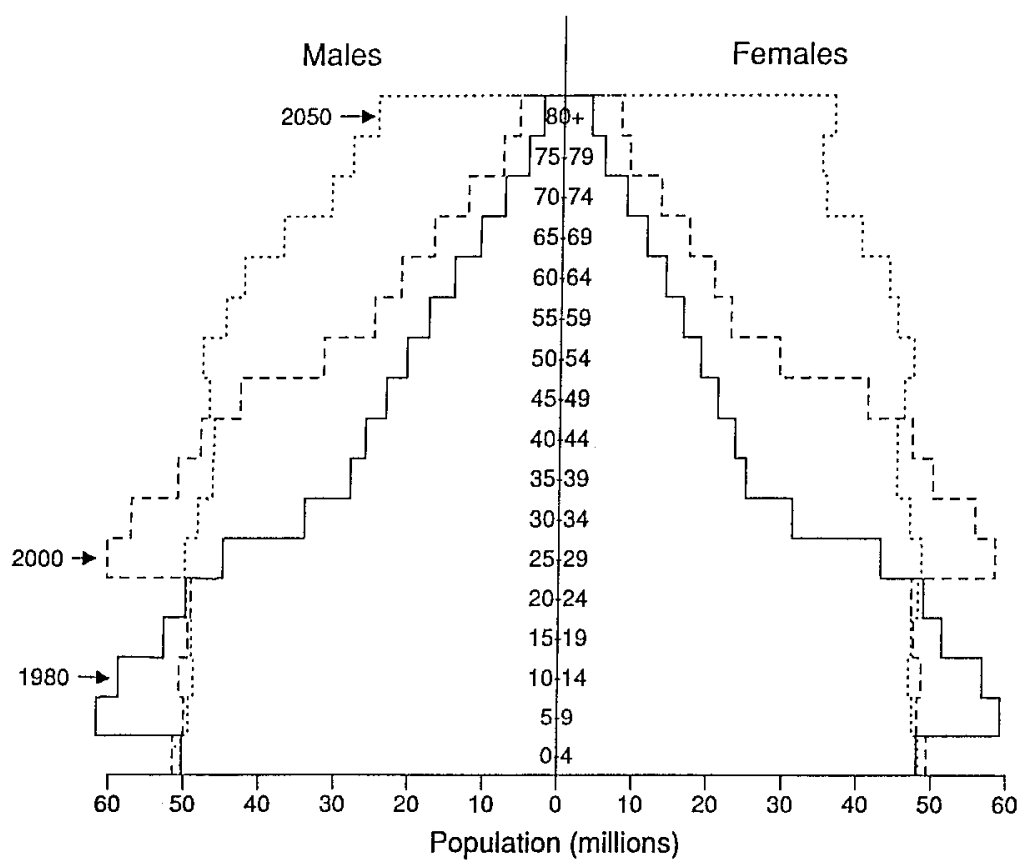

Figure 4. 
Figure 4 shows the population pyramids for the medium projection series for the years 1980,2000 and 2050 . This figure illustrates how the relatively large birth cohorts of the 1960s and early 1970s will continue to produce bulges in China's future age composition. This will occur not only as these cohorts age, but also as they pass through their reproductive years and produce an echo effect of the baby boom period in which they were born. There is now an effort underway in China to smooth out these subsequent baby boom echoes or birth peaks (Wu 1984b, 1985; Zhao 1979). It is also apparent that like most developed nations, the shape of the age pyramid in China will become much more rectangular as the population ages. Note that the largest difference in the age structures is at the apex where the old-old ( $80+$ ) population will grow faster than any other age group.

The medium-range projections of the population aged 0-14, 15-64, and 65 and over from 1980 to 2050 are located in Table A-II in the appendix. According to this table, the size of the population aged 0-14 will decline slightly between 1980 and 1990, and then remain fairly constant to the year 2050 . The population aged 15-64 will increase steadily to about the year 2020, and then level off. The 65 and older population is projected to increase throughout the projection time period where it is expected to account for about 18 percent of the total population by the year 2050 . These data indicate clearly that the projected changes in the actual size and distribution of the elderly population in China will be dramatic in the coming decades.

\section{TABLE II}

Projected percentage of the Chinese population at ages $0-14,15-64$, and $65+$,

low, medium and high assumptions (1990-2050)

\begin{tabular}{|c|c|c|c|c|c|c|c|c|c|}
\hline & & & & & S & Hawe & & & \\
\hline & & $65+$ & & & $0-14$ & & & $15-64$ & \\
\hline YEAR & $\begin{array}{c}\text { Low } \\
(1)\end{array}$ & $\begin{array}{l}\text { Medium } \\
\text { (2) }\end{array}$ & $\begin{array}{l}\text { High } \\
\text { (3) }\end{array}$ & $\begin{array}{r}\text { Low } \\
(4)\end{array}$ & $\begin{array}{l}\text { Medium } \\
\text { (5) }\end{array}$ & $\begin{array}{l}\text { High } \\
(6)\end{array}$ & $\begin{array}{r}\text { Low } \\
(7)\end{array}$ & $\begin{array}{l}\text { Medium } \\
\text { (8) }\end{array}$ & $\begin{array}{l}\text { High } \\
(9)\end{array}$ \\
\hline 1990 & 6.42 & 6.35 & 6.17 & 25.44 & 26.22 & 28.28 & 68.14 & 67.43 & 65.55 \\
\hline 1995 & 6.96 & 6.81 & 6.55 & 23.30 & 24.92 & 27.76 & 69.75 & 68.27 & 65.69 \\
\hline 2000 & 7.58 & 7.29 & 6.97 & 21.16 & 23.94 & 26.06 & 71.27 & 68.77 & 66.97 \\
\hline 2005 & 8.27 & 7.82 & 7.41 & 19.49 & 22.73 & 24.28 & 72.24 & 69.45 & 68.31 \\
\hline 2010 & 8.98 & 8.32 & 7.77 & 18.13 & 21.62 & 23.02 & 72.90 & 70.06 & 69.21 \\
\hline 2015 & 10.26 & 9.29 & 8.54 & 17.10 & 20.46 & 22.18 & 72.64 & 70.25 & 69.28 \\
\hline 2020 & 12.67 & 11.19 & 10.13 & 16.30 & 19.92 & 21.68 & 71.03 & 68.89 & 68.19 \\
\hline 2025 & 15.32 & 13.16 & 11.75 & 15.80 & 19.86 & 21.28 & 68.88 & 66.98 & 66.97 \\
\hline 2030 & 17.82 & 14.88 & 13.12 & 15.65 & 19.89 & 20.81 & 66.53 & 65.23 & 66.07 \\
\hline 2035 & 20.53 & 16.69 & 14.55 & 15.66 & 19.71 & 20.40 & 63.81 & 63.60 & 65.06 \\
\hline 2040 & 23.06 & 18.26 & 15.68 & 15.92 & 19.41 & 20.16 & 61.02 & 62.33 & 64.15 \\
\hline 2045 & 23.66 & 18.18 & 15.35 & 16.33 & 19.31 & 20.14 & 60.01 & 62.50 & 64.51 \\
\hline 2050 & 23.91 & 17.90 & 15.64 & 16.91 & 19.48 & 20.19 & 59.18 & 62.62 & 64.17 \\
\hline
\end{tabular}


Table II records the projected percentage distribution of the population in these age ranges using all three assumptions. The percentage of the total population aged 0-14 is projected to decline rapidly past the year 1980 because of the one-child policy, and then decline slightly to the year 2050 . The population aged 15-64 is projected to increase as a percentage of the total population until the year 2015, and then decline to a low of 62.3 percent in the year 2040. The 65 and older population is expected to increase as a proportion of the total population to between 15.6 to 23.9 percent by the year 2050 . These data indicate that regardless of which assumptions about fertility and mortality are used, the elderly population is expected to at least double, and more than likely triple as a proportion of the total population by the middle of the next century.

TABLE III

Projected growth rates of the population aged $65+$ and $80+$ in China from 1980 to 2050 (medium assumption)

\begin{tabular}{lcccccc}
\hline & \multicolumn{3}{c}{ Growth Rate (65t) } & \multicolumn{3}{c}{ Growth Rate (80+) } \\
\hline YEAR & $\begin{array}{c}\text { From 1980 } \\
(1)\end{array}$ & $\begin{array}{c}\text { Five-Year } \\
(2)\end{array}$ & $\begin{array}{c}\text { Annual } \\
(3)\end{array}$ & $\begin{array}{l}\text { From 1980 } \\
(4)\end{array}$ & $\begin{array}{l}\text { Five-Year } \\
(5)\end{array}$ & $\begin{array}{c}\text { Annual } \\
(6)\end{array}$ \\
\cline { 2 - 7 } & & & & & & \\
1985 & 13.81 & 13.81 & 2.59 & 27.55 & 27.55 & 4.87 \\
1990 & 29.00 & 13.35 & 2.51 & 53.36 & 20.24 & 3.69 \\
1995 & 45.85 & 13.06 & 2.46 & 79.94 & 19.33 & 3.20 \\
2000 & 64.20 & 12.59 & 2.37 & 110.78 & 17.14 & 3.16 \\
2005 & 83.65 & 11.84 & 2.24 & 144.30 & 15.90 & 2.95 \\
2010 & 102.24 & 10.12 & 1.93 & 179.78 & 14.52 & 2.71 \\
2015 & 132.57 & 15.00 & 2.79 & 217.88 & 13.62 & 2.55 \\
2020 & 187.68 & 23.69 & 4.25 & 259.78 & 13.18 & 2.48 \\
2025 & 246.31 & 20.38 & 3.71 & 297.65 & 10.52 & 2.00 \\
2030 & 298.29 & 15.01 & 2.80 & 378.30 & 20.28 & 3.69 \\
2035 & 350.81 & 13.19 & 2.48 & 531.48 & 32.03 & 5.56 \\
2040 & 394.57 & 9.71 & 1.85 & 656.13 & 19.74 & 3.60 \\
2045 & 392.43 & -0.43 & -0.09 & 743.56 & 11.56 & 2.19 \\
2050 & 383.94 & -1.73 & -0.35 & 845.85 & 12.13 & 2.29 \\
\hline
\end{tabular}

Another way of illustrating the growth of the elderly population is to examine the projected growth rates of the population in selected age ranges. Table III shows the growth rates of the Chinese population aged 65+ and 80+ from 1980 to the year 2050 in five-year intervals using the medium-range assumptions. The numbers listed in columns 1 and 4 show the projected cumulative growth rates of the population in these age groups from 1980. For example, the population aged $65+$ is projected to grow by 13.81 percent from 1980 to 1985 , and by 246 percent by 2025. The numbers listed in columns 2 and 5 show the projected five-year growth rates of the population within each five-year period. The figure of 12.13 in column 5 for the year 2050 means that the population aged $80+$ is 
projected to increase by 12.13 percent from 2045 to 2050 . The numbers listed in column 3 and 6 show the projected annual growth rates of the population within each 5-year time interval. The figure of 4.25 in column 3 for the year 2020 means that the population aged $65+$ is projected to increase by 4.25 percent annually from 1980 to 1985 . These data illustrate that the population aged 65 and over will reach its peak growth rate from the observed 1980 population in the year 2040, after which slight declines are expected. The most rapid annual and 5-year growth rates for this age group will occur between the years 2020 and 2030. Even more striking is the projected growth rate of over 845 percent for the population aged $80+$ from 1980 to the year 2050. The largest annual and 5 -year growth rates will occur between 2030 and 2045 for this age group. The timing of these peak growth rate years are directly related to the aging of the large birth cohorts born during the period from 1960 to 1970. It is apparent from these data that not only will the Chinese population age, but the size of the elderly population (particularly the oldest old) will grow at an extremely rapid pace before the year 2050 .

The measures of population aging are also projected to increase rapidly in China over the next 70 years, and some of these measures are quite sensitive to the various assumptions used. Table IV lists both observed and projected measures of population aging using all three projection assumptions for the years 2000,2025 , and 2050 . Note that for the year 2000 , most of the measures listed here are not sensitive to the various projection assumptions with the exception of those measures which include the population aged $0-14$. This occurs because these measures are heavily dependent on differences in assúmptions about fertility, and these did vary considerably to the year 2000 (see Table A-I).

By the year 2025 all of the other measures listed here, with the exception of those which evaluate the aging of the elderly, become sensitive to the various assumptions, not only because of earlier differences in assumptions about fertility, but also because the different mortality assumptions begin to alter significantly the projected size of the elderly population. By the year 2050 virtually all of the measures of population aging show variation with the alternative fertility and mortality assumptions.

These prospective trends in population aging in China have been noted in numerous other publications. While there are differences in the assumptions about how fertility and mortality might change in the future, the consensus is that population aging will occur in China at an accelerated pace by comparison to other nations of the developed and developing world. Minor variations in assumptions about fertility will tend to alter projections of the proportional distribution of the elderly population while minor variations in assumptions about mortality will influence the size of the elderly population. What has yet to be revealed by other researchers in their projections about population aging in China (or any other country) is the relative contribution of the various components of population change to this process. The importance of decomposing 


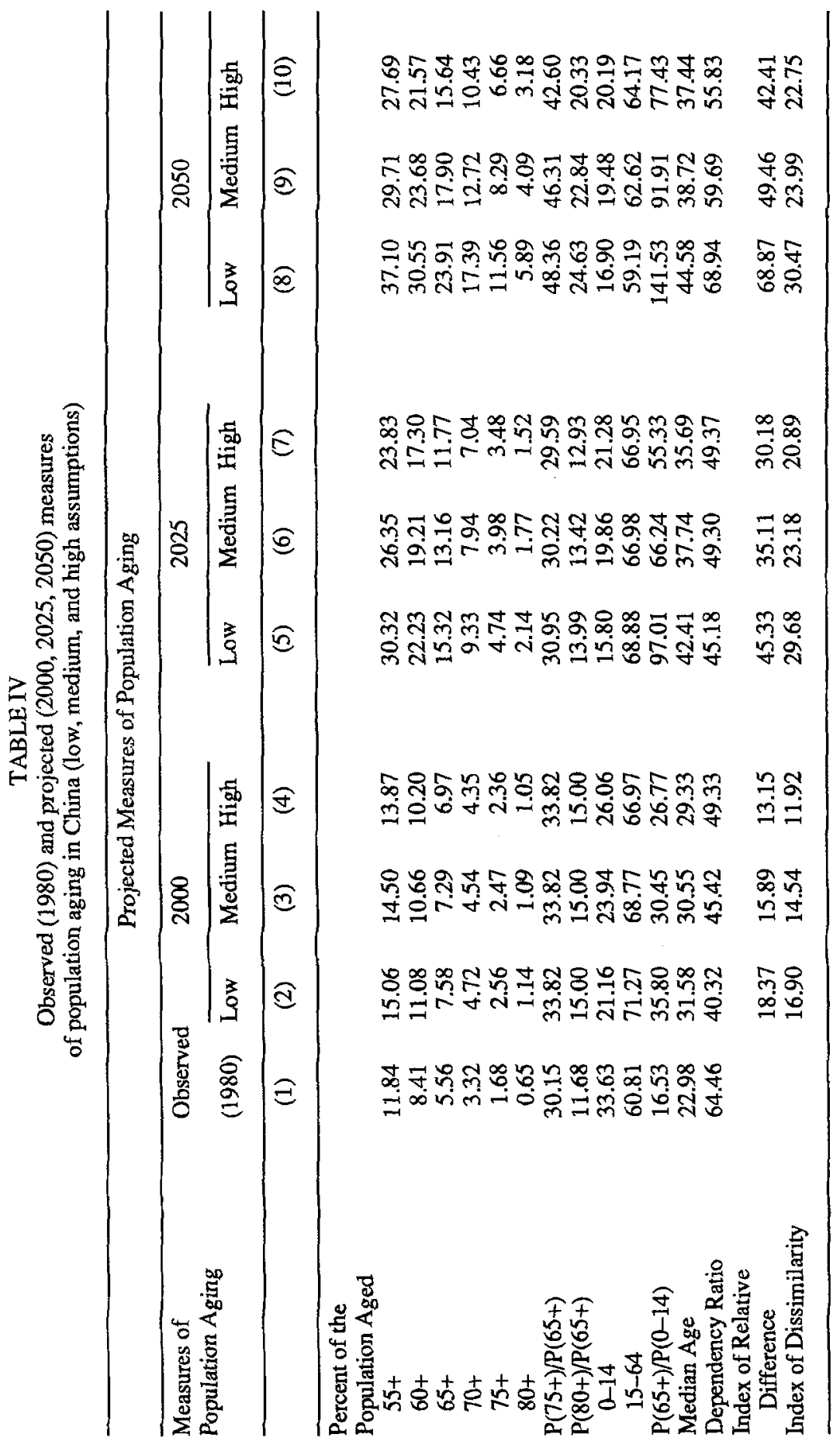


the aging process into its relative components is that it then becomes possible to determine 1) just how much momentum for aging is built into the age structure and present vital rates and thus how much of the aging process is inevitable, and 2) precisely what kinds of demographic policies might be implemented to alter vital rates so as to minimize the problems of population aging. In the following section the projected measures of population aging are decomposed into the relative contributions of each of the components which will influence its course.

\section{DECOMPOSING MEASURES OF POPULATION AGING IN CHINA}

The age structure of a closed population like China can change as fertility and mortality change. Typically, mortality transitions do not occur uniformly at all ages, as they initially tend to favor the youngest age groups as a result of a reduced risk of infectious and parasitic diseases, and later they affect the middle and older age groups as the risk of degenerative diseases tend to subside. The mortality declines in China from 1953 to 1964 favored the youngest age groups, making the population younger as the base of the age pyramid expanded rapidly. Projected declines in mortality beyond 1985 are expected to favor the middle and older age groups, and will contribute to population aging by expanding the middle and apex of the age pyramid.

As fertility rates decline from high to low levels, a population ages rapidly (as measured by proportional changes in age composition) because the relative size of the youngest (and largest) age groups decline rapidly. Conversely, increases in fertility will make a population younger.

Even in the absence of changes in fertility and mortality, a population can age as a result of the momentum that is inherent in its initial age structure and initial vital rates. This momentum is analogous to the momentum for population growth that may persist in a population with a young age structure even after fertility has reached replacement levels. For example, the fertility and mortality rates that are present in a population at any moment in time are likely to differ significantly from those that prevailed during the previous 80 years which created the age structure at that moment. This is the case with China. The relatively large cohorts born during the 1960s and early 1970s will shape the age structure of China's population in the future, just as the baby boom cohort of 1946-1964 will continue to affect the U.S. population age structure well into the next century. This concept of momentum for population aging is what led several Chinese scientists to conclude that population aging would not be a problem in China because there was nothing that could be done to alter the course of past demographic events (Hao and Wang 1987; Hu et al. 1980; Ma 1987). What these scientists failed to appreciate was that four demographic factors regulate the rate at which a population ages from any moment in time: the age structure at any given time - referred to here as time zero (e.g. the cumulative effects of past levels of fertility and mortality), the vital rates at time zero, and prospective changes in fertility and mortality. Projected changes in the 
measures of population aging may therefore be decomposed into the relative contributions that are made by each of these components. A method for performing such a decomposition has been developed by Grigsby (1988).

The decomposition method developed by Grigsby (1988) is similar to a method used by $\mathrm{Yu}$ and Horiuchi (1987), with one notable difference. Both methods develop a series of four projection scenarios which represent the four different combinations of either altering, or holding constant, assumptions about fertility and mortality. These include fertility constant and mortality constant (FCMC); fertility constant and mortality varying (FCMV); fertility varying and mortality constant (FVMC); and fertility varying and mortality varying (FVMV). By considering differences in population growth that occur in different age groups under these various scenarios, it is possible to determine the precise contribution of each component to projected changes on the measures of population aging.

The first projection scenario (FCMC) assumes that the vital rates observed at time zero for each calendar year continue to the end of the projection period. In this case time zero is the year 1980 in China (United Nations 1986), and the projection time frame is 70 years (to the year 2050). The fourth projection scenario (FVMV) refers to a series or range of assumptions about how fertility and mortality rates will change over the projection time frame. The second and third projection scenarios vary either fertility or mortality while holding the other constant.

The effect of the initial conditions (e.g., fertility and mortality conditions that prevail at time zero) on prospective trends in measures of population aging is determined by comparing the difference between the age structure at time zero and the age structure projected under the FCMC scenario. It is at this point that the Grigsby (1988) and Yu and Horiuchi (1987) methods differ. Yu and Horiuchi (1987) labeled this difference as the effect of the initial age structure, and defined it only as the fertility and mortality schedules that prevailed at time zero. By attributing subsequent changes in population aging solely to the fertility and mortality schedules at time zero, the effects of past trends in fertility and mortality which created the age structure at that time are ignored (Coale 1987). Such a definition would be appropriate only if the fertility and mortality conditions at time zero are the same as those which produced the age pyramid for that year. In China, as with all other nations, the fertility and mortality conditions of the past 80 years are far different from those which prevail today. Continuing the 1980 vital rates would produce a different age structure, one that in the short-run would depend on both the current age structure and vital rates, but in the long-run would depend only on the fertility and mortality schedules at time zero (Coale 1964; Horiuchi and Preston 1988). The method developed by Grigsby (1988) separates the effects of both age composition and vital rates at time zero on measures of population aging.

The effect of prospective changes in fertility rates on measures of population aging is assessed by comparing the FCMV projection with the FCMC projection 
scenario, and the FVMV projection with the FCMV scenario. This second component may be thought of as the interaction of changing fertility and mortality. With this methodology the effect of changing fertility on measures of population aging is computed as the average of these two components (see $\mathrm{Yu}$ and Horiuchi 1987). The effect of changing mortality on measures of population aging is computed analogously. The main advantage of defining the components of demographic change in this manner is that they sum exactly to the total change in the measure of population aging, which is the difference between the measure observed in 1980 and that observed under the FVMV projection scenario.

Table $\mathrm{V}$ shows the results of this decomposition in terms of the percentage contribution of each of the components of population change to the projected total changes in the measures of population aging from 1980 to 2050 (medium assumption). The data in column 1 show the total projected change over the 70 year time frame. The figure of 17.87 in column 1 for the population aged 55+ means that from 1980 to 2050 the population in this range is projected to increase by 17.87 percent over what was observed in 1980. Of that increase, 24.8 percent and 10.4 percent is attributable to prospective changes in fertility and mortality, respectively; 65.9 percent will be attributable to the fertility and mortality schedules present in 1980; while the age structure present in 1980 (which is a function of past trends in fertility and mortality) would actually have a negative effect on the projected change of 1.1 percent.

These data suggest that at least with respect to the percentage of the population at various ages over the age of 55 , the contribution of prospective changes in mortality becomes increasingly important as older age groups are considered. For example, for the 55+ population 10.4 percent of the projected change in this measure from 1980 to 2050 will be attributable to mortality change while 28.7 percent of the projected change will be attributable to mortality for the $80+$ population. Prospective changes in fertility will contribute on average about one fourth of the change in these measures. The effects of the initial age structure and fertility and mortality rates at time zero are at least 50 percent for each of these measures. Therefore more than half of the prospective changes in these measures of population aging are inevitable and not subject to change by altering either fertility or mortality patterns.

In summary, prospective changes in the aging of the elderly population will be largely influenced by the present age structure of China (or past levels of fertility and mortality), and vital rates that China has already achieved. Future changes in mortality will contribute from 26.7 percent to 34.5 percent of the aging of the elderly population itself, while changes in fertility will not contribute at all because the population that will be in these age groups between now and the year 2050 have already been born. All of the other measures of population aging listed in Table $\mathrm{V}$ will be influenced more by past and present levels of fertility and mortality than by future changes in these vital rates. 


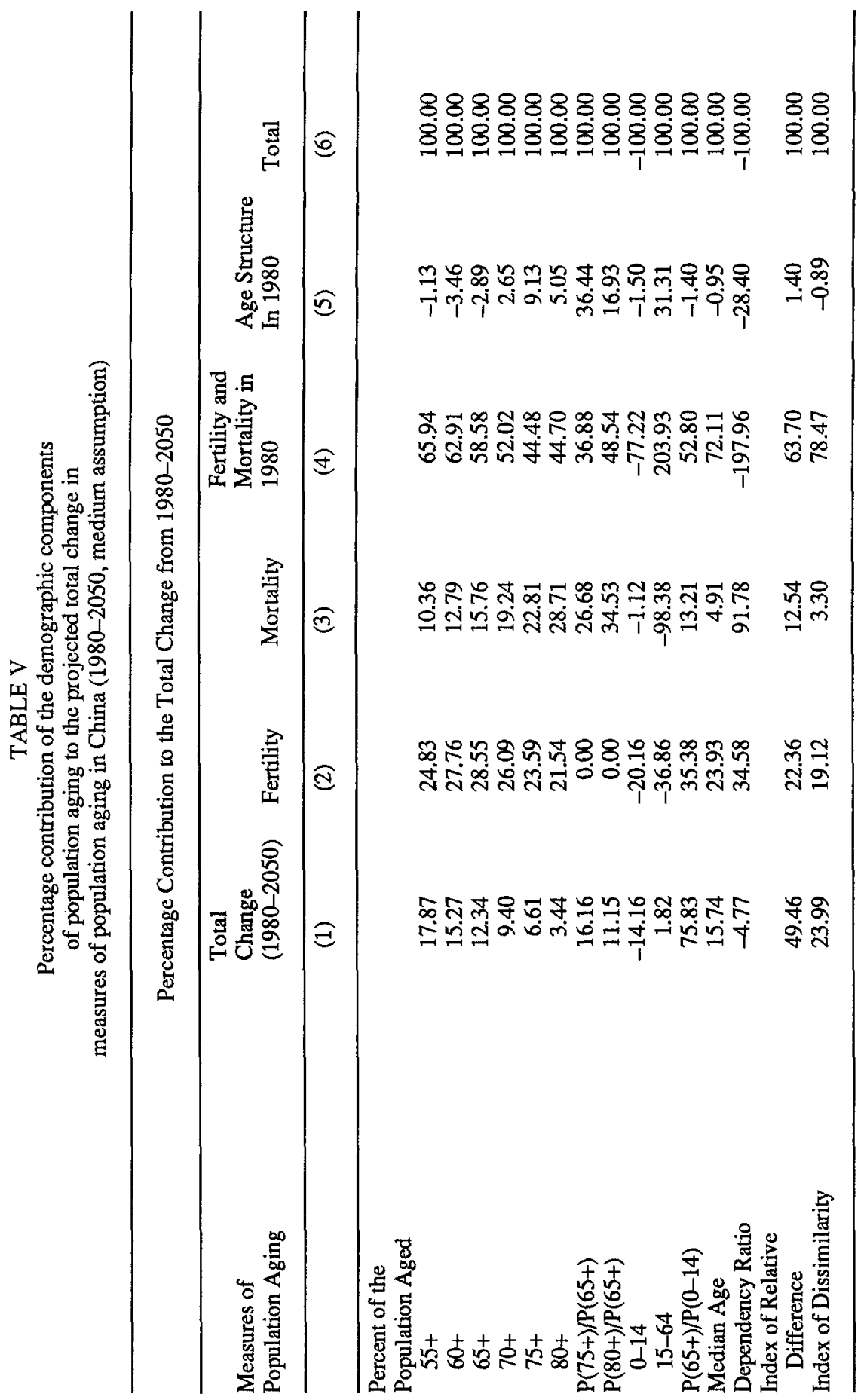




\section{CONCLUSIONS}

Based on the projections presented here, the process of population aging in China will accelerate into the 21st century. Aging will occur primarily as a result of past trends in fertility and mortality and current vital rates, both of which have created a momentum for population aging that cannot be reversed. Recent trends toward declining fertility and mortality will further fuel the process of population aging. The relative proportions of the total population that are elderly will increase, the absolute size of the elderly population will grow rapidly, and the elderly population itself will age. The magnitude of the increase in the size of the elderly population is probably the most dramatic consequence of this aging phenomenon, and it may be put in perspective by noting that there will be more people over the age of 65 in China in the year 2050 than there are people of all ages in the United States today.

Current family planning policies in China are designed to reduce birth rates and the overall rate of population growth. If this policy is successful in reducing fertility rates much below replacement levels and sustaining them even for a short time period, the base of the age structure will narrow, bringing even more rapid acceleration of population aging. A relaxation of the one-child policy at selected time periods during the next 70 years could smooth the birth peaks that are expected from the echo effects of previous baby booms, and it would decelerate population aging by reducing imbalances in the age composition that are anticipated in the future.

Mortality declines that have occurred in China in recent decades have occurred at all ages, but have tended to favor the young over the old. They occurred at an extremely rapid pace and brought forth prospects for survival in China that rival that of most developed nations (Banister and Preston 1981). While these mortality declines tended to decelerate population aging between 1953 and 1964 by allowing larger proportions of younger birth cohorts to survive past the first year of life, past mortality declines which benefitted younger age groups will subsequently add to population aging in the future by increasing the size of cohorts that will survive to older ages. This will tend to accelerate the rate at which the population ages as measured by the size and growth rate of the elderly population and the aging of the elderly population itself.

In this study it is assumed that male life expectancy at birth will increase from 1980 to the year 2050 by anywhere from 4.0 to 9.9 years, and female life expectancy at birth is projected to increase from 5.5 to 11.1 years. Since these projected levels of life expectancy for China for the year 2050 are comparable to that of the United States today, this places China approximately 70 years behind the U.S. with respect to mortality. Given that 1) recent mortality declines in China have propelled life expectancy within an extremely short time period to levels that are comparable with developed countries, 2) the Chinese population already maintain lifestyles that are favorable toward longevity in terms of diet 
and exercise, and 3) there appears to be a tremendous potential for further reductions in mortality throughout the age structure even with minor advances in medical technology or the distribution of health care to rural areas, it is quite possible that the low mortality assumptions used here and in other studies may be underestimating China's potential for longevity. If mortality declines are underestimated for China, the size and proportional distribution of the elderly, particularly the oldest-old, will be considerably higher than current estimates indicate. For example, if life expectancy at birth were to increase to 85 for females and 80.5 for males by the year 2050 in China, the projected size of the 65 and older population would be 339.6 million people - or 48.4 million more people in this age group than is currently projected in this study under the low mortality assumption. This difference of 48.4 million people in the 65 and older age group occurs with only a .07 per year difference in the projected life expectancy at birth. Additionally, under these more favorable mortality conditions the number of people projected to be age 80 and over in China in the year 2050 would exceed 95 million persons.

Based on the data presented here and in other studies, it is apparent that population aging will progress in China at a pace that is unmatched in the rest of the world. While scientists throughout the world have been watching the development of policy associated with population growth and the one-child family policy in China with great interest, it is likely that issues associated with population aging will soon dominate their attention. The success of China's strict one-child policy indicates that the Chinese government is quite capable of rapidly altering internal demographic conditions. While the data presented here indicate that at least 50 percent of the aging process in China during the next 70 years is inevitable, it is still possible to take measures today to reduce the rate of population aging and dampen fluctuations in the age structure while preparing for the economic and social conditions which accompany an aging population. The question that remains is what kinds of policies will the Chinese adopt to address some of these problems.

\section{ACKNOWLEDGEMENTS}

This paper was presented at the IUSSP seminar on "Mortality Transition in South and East Asia" held in Beijing, China, August 29-September 2, 1988. An earlier version was presented at the annual meetings of the Gerontological Society of America held in Washington, D.C., November 19-22, 1987. Dr. Grigsby's research support was provided in part by a postdoctoral training grant from the National Institute on Aging (1-T32-AG00151-01). Dr. Olshansky's research support was provided by National Institute on Aging Grant No. AG06996-01. The authors wish to thank Alberto Polloni and Zeng Yi for comments on an earlier draft of the manuscript, and Lee Jay Cho and Andrew Mason at the East-West Population Institute, Honolulu, Hawaii, for providing the authors with the opportunity to travel to China through their Summer 
Seminar program. The authors also wish to thank Judith Banister for providing us access to her files on China.

Please address correspondence to S. Jay Olshansky, Ph.D., Center on Aging, Health, and Society, Department of Medicine, University of Chicago, $5841 \mathrm{~S}$. Maryland, Box 72, Chicago, Illinois, 60637.

\section{NOTES}

${ }^{1}$ Many of the discussions of the scientific and political aspects of Chinese demography appear in the popular literature such as newspaper articles or Foreign Broadcast Information Service publications. Very few articles have appeared in the refereed journals, particularly those discussions of policy associated with China's family planning program. While many of these articles which appear in the popular literature tend to present the Chinese government's view on these demographic issues, there are many instances in which the debates which tend to surface between scientists, appeared in these publications. Also, the reports of family planning and optimum population targets set forth by political officials in these publications are believed to be accurate because these are cleared by government officials before release. Many of the references to Chinese authors listed here are based on English translations of these publications, and they are available to the reader upon request.

2 These goals or targets were set forth at different times by members of a Central Work Conference in Beijing, by members of the National Pcople's Congress, at several times by Vice Premier Chen Muhua, and by Vice Premier Yao Yilin. For more details about these targets see references 9-14 in Aird (1986).

${ }^{3}$ These negative sanctions included the withdrawal of benefits that are offered to families that have only one child. These benefits varied for different cities and by rural/urban residence, but generally included a monthly child health care fee, priority for entering nurseries and kindergartens, free tuition for the child when they enter elementary and secondary schools, preference for housing allotments in urban areas and plot distribution in rural areas, and an additional 5 percent above what would ordinarily be given in the form of a pension after retirement for parents who have only one child (WHP 1979a; WHP 1979b). Upon the birth of a second child all benefits would stop and all monetary benefits previously received would have to be paid back in installments.

${ }^{4}$ The year 2020 was viewed as critical because the large baby boom cohorts of the $1960 \mathrm{~s}$ would begin to reach the age of 60 in that year.

5 Chinese scientists attempted early on to disassociate population aging from their onechild policy by arguing that the former is dependent exclusively on past trends in fertility and mortality and the shape of the present age composition. The rationale here is based on the assumption that population aging is inevitable because of past mistakes in controlling births, and that between now and the year 2020 there is plenty of time to plan for any population aging problems that may arise. Others argued that a successful family planning program would address both population growth and population aging by reducing growth rates today, and by reducing the size of the population that would survive to older ages in the future. The disassociation between population aging and the one-child policy occurred primarily because declining birth rates are known to cause an acceleration of population aging, and the recognition of this fact might have led some to waver in their support for the family planning effort.

6 The importance of the mortality assumption to forecasts of the size of China's elderly population has been discussed by Xu (1985).

7 Banister (1988) projected that life expectancy at birth in China would increase from an overall level of 66 in 1982 (which was an average of male and female life expectancy at birth) to 81 in the year 2050 . Since these forecasts of life expectancy were made for the 
country as a whole, sex differentials in mortality and life expectancy were not explicitly taken into consideration. As a result, the projected life expectancy of males is considerably higher than what has been projected in this study. In the forecasts presented here three separate sets of assumptions were made about life expectancy at birth, by sex (see Table A-I). Since our medium-range forecasts of life expectancy at birth for males and females in China in the year 2050 were 73.1 and 77.6, respectively, this difference in the mortality assumption accounts for all of the 73.1 million person difference in the forecasts of the 65 and older population. It is therefore apparent that, at least with regard to the projected size of the elderly population in China, the mortality assumption is of critical importance.

${ }^{8}$ The United Nations defines a population that has aged as one that has at least $7 \%$ of its population aged 65 and over. This definition has been used by Chinese scientists to point out that China has yet to be defined as "old" by international standards, and as a result population aging need not be considered a problem at this time. It should be noted that the figure of $7 \%$ is an arbitrarily chosen number that has no intrinsic meaning. A country with a greater or lesser percentage of its total population aged 65 and older may be conisidered an aged or aging population, and what is really more important are the growth rates and changes in the absolute size of the elderly population. For instance, while the percentage of China's total population that was aged 65 and older increased from $3.56 \%$ to $4.91 \%$ from 1964 to 1982 , the absolute size of this age group doubled during this same time period. While China had yet to reach the international definition of an aged population by 1982 , the fact is that the population had aged rapidly during that 18 year time period.

9 The childless elderly population in China are supported by the "five guarantee" system which provides state-funded food, clothing, medical care, housing, and burial expenses.

${ }^{10}$ China is considered a closed population so migration is not considered a component which may influence population aging for the country as a whole. However, internal migration patterns might influence the rate of population aging within certain rural and urban areas.

\section{REFERENCES}

Aird, J. 1986 Future Implications of Alternative Family Planning Policies in China. Center for International Research, U.S. Bureau of the Census, Washington, D.C.

Banister, J. 1986 China: Recent Trends in Health and Mortality. Center for International Research, Bureau of the Census.

Banister, J. 1987 China's Changing Population. Stanford University Press, Stanford, California.

Banister, J. 1988 Implications of the Aging of China's Population. Center for International Research, Bureau of the Census, Washington, D.C.

Banister, J., and S. Preston, 1981 Mortality in China. Population and Development Review, 7(1):98-110.

Bongaarts, J., and S. Greenhalgh 1985 An Alternative to the One-Child Policy in China. Population and Development Review 11(4):585-617.

Chen Muhua 1979a (For the Realization of the Four Modernizations, There Must be Planned Control of Population Growth), JMJP, August 11, 1979, p. 2 (English translation).

Chen Muhua 1979b "Chen Muhua Outlines Significance of Planned Parenthood." FBIS, No. 251, December 28, pp. L7-9.

China Population Newsletter 1986 "Minister Wang Wei on Family Planning Policy and Population Aging. Volume 3, No. 4, pp. 1-4.

Coale, A.J. 1964 "How a Population Ages or Grows Younger" in Populations: The Vital Revolution, ed. by R. Freedman, New York: Doubleday. 
Coale, A.J. 1981 Population Trends, Population Policy, and Population Studies in China. Population and Development Review, 7(1):85-97.

Coale, A.J. 1987 Personal communication.

The Futures Group. 1987 Demproj. Version 2.62. Glastonbury, Ct.: The Futures Group.

Foreign Broadcast Information Service 1980 "CCP Central Committee Open Letter on Population Control." No. 189, September 26, p. L1.

Grigsby, J. 1988 The Demographic Components of Population Aging. Pomona College. Manuscript under review.

Guanming Daily 1979 (The Strategic Task of Population Control Must be Accomplished), GMRB, Beijing, June 30, pp. 1,3 (English translation).

Gui Shixun 1983 (Lecture VII: The Rate of Change of the Population Must Be Favorable to Social Development). Shehui (Society), No. 4, August 20, pp. 61-65 (English translation).

Hao Maishou, Wang Laihua 1987 (A Brief Discussion on the Prospect of Population Aging in China). Tianjin Social Sciences, No. 3, June 15, pp. 52-55 (English translation).

Hermalin, A.I. 1966 The Effect of Changes in Mortality Rates on Population Growth and Age Distribution in the United States. Milbank Memoral Fund Quarterly, 44(4):451-469.

Horiuchi, S., and S. Preston 1988 Age-Specific Growth Rates: The Legacy of Past Population Dynamics. Demography 25(3):429-441.

Hsiao, W.C. 1988 The Incomplete Revolution: China's Health Care System Under Market Socialism. Paper presented at the Fairbank Center for East Asian Research, May 13-15.

Hu Baosheng, Wang Yingzhi, Wang Wancheng, and Jiang Zhenghua 1980 (Fully Understand the Nature of Planned Population Control). RMRB, May 27, p. 5 (English translation).

Keyfitz, N. 1984 The Population of China. Scientific American, 250(2):38-47.

Kinsella, K. 1988 Aging in the Third World. CIR Staff Paper No. 35, Center for International Research, Bureau of the Census.

KMJP, 1979 (The CCP Kweichow Provincial Committee Circulates a Notice Throughout the Province. Two Prefecture and Hsien Leadership Cadres Who Failed to Practice Family Planning Have Received Severe Punishment), September 7, p. 2 (English translation).

Liang, J., Tu, E., Chen, X. 1986 Population Aging in the People's Republic of China. Social Science and Medicine, 23(12):1353-1362.

Ma, Y. 1987 Family Planning and Population Aging Have No Quantitative Relationship. RKYJ, No. 2, March 29, p. 59.

Ming Bao 1979 The Future Policies Decided by the Central Work Conference are Policies on Economic Readjustment and Not Contraction. Foreign Broadcast Information Service, No. 119, June 19, p. L14.

Nan Zhongji 1986 On Population Aging in China. China Population Newsletter, 3(4).

Neugarten, B.L. 1974 Age Groups in American Society and the Rise of the Young-Old. Annals of the American Academy of Political and Social Science, September, pp. 187-198.

NFRB 1983 (Vice Governor Wang Pingshan Answers Reporter on the Questions Concerning Policies on Birth Control Techniques and Childbearing), May 15, p. 2 (English translation).

Qiu Jianwei 1984 (To Recognize Fully the Importance and the Urgency of China's Population Aging Problem). RKYJJ, No. 6, December 25, pp. 28-33 (English translation).

Shryock, H.S, and J.S. Siegel 1980 The Methods and Materials of Demography. Washington, D.C.: U.S. Government Printing Office. 
Song Jian, Ti'an Xueyuan, Li Guangyuan, Yu Jingyuan 1985 Scientists Predict Various Rates of Future Population Growth. Reported by Yu Zhenpeng, Foreign Broadcast Information Service, No. 033, February 15, pp. L11-13.

Tian Xueyuan 1980 On the Question of the Growing Percentage of Old People. Foreign Broadcast Information Service, No. 83, May 14, p. 67.

Tian Xueyuan 1984 On Changes in the Age Composition of the Population and Policy Options for Population Planning. Social Sciences in China No. 3, pp. 191-206.

Tao Liqun 1984 (The Problem of Aging of the Chinese Population), Society No. 2, April 20, pp. 107 (English translation).

United Nations 1982 Demographic Indicators of Countries: Estimates and Projections as Assessed in 1980. New York: United Nations.

United Nations 1986 World Population Prospects: Estimates and Projections as Assessed in 1984. New York: United Nations.

U.S. Bureau of the Census 1988 Statistical Abstract of the United States, U.S. Department of Commerce, U.S. Government Printing Office, Washington, D.C.

Wei H. 1987 Growth of the Aged Population in China: Trends and Policy. In Aging China: Family, Economics and Governments Policies in Transition. J.H. Schultz and D. Davis-Friedman, eds. Washington, D.C.: The Gerontological Society of America.

WHP 1979a (Several Regulations of the Shanghai Municipal Revolutionary Committee Concerning the Promotion of Family Planning). Family planning regulations passed by the Fifth Plenary Session of the Shanghai Municipal Revolutionary Committee, August 22, p. 2 (English translation).

WHP 1979b (Population Control is a Strategic Task of Importance), June 30, p. 1 (English translation).

Wu, Yuanjin and Xu, Qin 1987 The Impact of an Aging Population on Socio-Economic Development and Families in China. In Aging China: Family, Economics and Government Policies in Transition, J.H. Schultz and D. Davis-Friedman, eds. Washington, D.C.: The Gerontological Society of America.

Wu Changping 1984a Problems of Old Age and Our Countermeasures. FBIS, JPRS-CPS-84-065, October 2, pp. 24-28.

Wu Changping 1984b (The Two Problems Which We Should Pay Attention To). Economic Digest, No. 4, p. 58 (English translation).

Wu Changping 1985 The Development Strategy Has to Be Based on Our Population Problem. JPRS-CPS-85-041, May 7, pp. 56-66.

Wu, I. 1986 Aging in a Changing Population, China in the Twenty-First Century. Unpublished manuscript.

Xu Qin 1985 Analysis of the Aging Process and Characteristics of China's Population. Renkou Yanjiu (Population Research) No. 6, November 29, pp. 28-30 in FBIS, JPRS-CEA-86-080, June 25, 1986.

Yu, Yuen-Chung and S. Horiuchi 1987 Population Aging and Juvenation in Major Regions of the World. Paper presented at the 1987 Annual Meetings of the Population Assocation of America, Chicago, Illinois.

Zhao, L., and C. Jiang 1986 (Some Thoughts on the Problem of Population Aging), JJWT (Problems of Economy), No. 12, pp. 58-59 (English translation).

Zhao, G. 1979 (The Promotion of the One-Child Couple is an Effective Way of Levelling Birth Peaks). Sichuan University Journal, Philosophy and Social Sciences Edition, Serial Monograph No. 3, pp. 40-45 (English translation).

\section{University of Michigan and Pomona College}

Center on Aging, Health and Society and National Opinion Research Center, University of Chicago 
APPENDIX

TABLE A-I

Fertility and Mortality Projection Assumptions for China (1980-2050)

\begin{tabular}{|c|c|c|c|c|c|c|c|c|c|}
\hline & \multicolumn{3}{|c|}{$\begin{array}{c}\text { Total } \\
\text { Fertility Rate }\end{array}$} & \multicolumn{3}{|c|}{$\begin{array}{c}\text { Male } \\
\text { Life Expectancy }{ }^{\mathbf{2}}\end{array}$} & \multicolumn{3}{|c|}{$\begin{array}{c}\text { Female } \\
\text { Life Expectancy } \\
\end{array}$} \\
\hline & Low & Medium & High & Low & Medium & High & Low & Medium & High \\
\hline Year & (1) & (2) & (3) & (4) & (5) & (6) & (7) & (8) & (9) \\
\hline $1980-85$ & 2.30 & 2.36 & 2.80 & 66.7 & 66.7 & 66.7 & 68.9 & 68.9 & 68.9 \\
\hline $1985-90$ & 1.90 & 2.11 & 2.50 & 68.0 & 68.0 & 68.0 & 70.9 & 70.9 & 70.9 \\
\hline $1990-95$ & 1.65 & 1.91 & 2.20 & 69.0 & 69.0 & 69.0 & 72.6 & 72.6 & 72.6 \\
\hline $1995-00$ & 1.50 & 1.91 & 2.10 & 70.0 & 70.0 & 70.0 & 73.9 & 73.9 & 73.9 \\
\hline $2000-05$ & 1.52 & 1.91 & 2.10 & 70.7 & 70.4 & 70.1 & 75.1 & 74.6 & 74.0 \\
\hline $2005-10$ & 1.55 & 1.95 & 2.10 & 71.4 & 70.8 & 70.1 & 76.1 & 75.1 & 74.1 \\
\hline $2010-15$ & 1.57 & 1.98 & 2.10 & 72.1 & 71.2 & 70.2 & 77.1 & 75.7 & 74.3 \\
\hline $2015-20$ & 1.64 & 2.05 & 2.10 & 72.6 & 71.5 & 70.3 & 77.9 & 76.2 & 74.4 \\
\hline $2020-25$ & 1.70 & 2.11 & 2.11 & 73.0 & 71.7 & 70.4 & 78.6 & 76.6 & 74.5 \\
\hline $2025-30$ & 1.78 & 2.11 & 2.11 & 73.7 & 72.1 & 70.4 & 78.9 & 76.8 & 74.6 \\
\hline $2030-35$ & 1.86 & 2.11 & 2.11 & 74.4 & 72.5 & 70.5 & 79.2 & 77.0 & 74.7 \\
\hline $2035-40$ & 1.95 & 2.11 & 2.11 & 75.2 & 72.9 & 70.6 & 79.4 & 77.2 & 74.9 \\
\hline $2040-45$ & 2.03 & 2.11 & 2.11 & 75.9 & 73.3 & 70.6 & 79.7 & 77.4 & 75.0 \\
\hline $2045-50$ & 2.11 & 2.11 & 2.11 & 76.6 & 73.1 & 70.7 & 80.0 & 77.6 & 75.1 \\
\hline
\end{tabular}

a The mortality projection assumptions are presented here in summary form as life expectancy at birth. The low mortality assumptions (columns 4 and 7 ) produce the highest life expectancy and the high mortality assumptions (columns 6 and 9) produce the lowest life expectancy. Coale-Demeny west model life tables were used for projecting mortality from life expectancy at birth.

It should be noted that the model life tables developed originally by Dr. Coale and Dr. Demeny are currently under revision. When completed, the revised west model life tables will contain more favourable mortality schedules and survival patterns than is currently the case. If the population projections presented here were re-calculated using these new model life tables, the size and proportional distribution of China's elderly population would be even greater than what is projected here.

Source: Fertility projection assumptions from 1980-2025 were drawn from United Nations estimates (1984). The high and medium fertility series are then projected to continue at 2.11 through the end of the projection period. The low fertility series is projected to increase linearly from 1.70 at the period $2020-25$ to 2.11 by $2045-50$. The low mortality projection assumptions from 1980-2025 were drawn from estimates made by the United Nations (1984) which was their only mortality assumption for China. From 2025-2050 the low mortality parameters are projected to increase linearly to a life expectancy of 80.0 years for females and 76.6 years for males. The high mortality series is identical to the low mortality series from 1980-2000, and then life expectancy is projected to increase linearly to a life expectancy of 75.1 for females and 70.7 for males by the period $2045-50$. These values are reached in the low mortality series by the period $2000-05$. The medium mortality projection assumptions are an average of the high and low assumptions. 


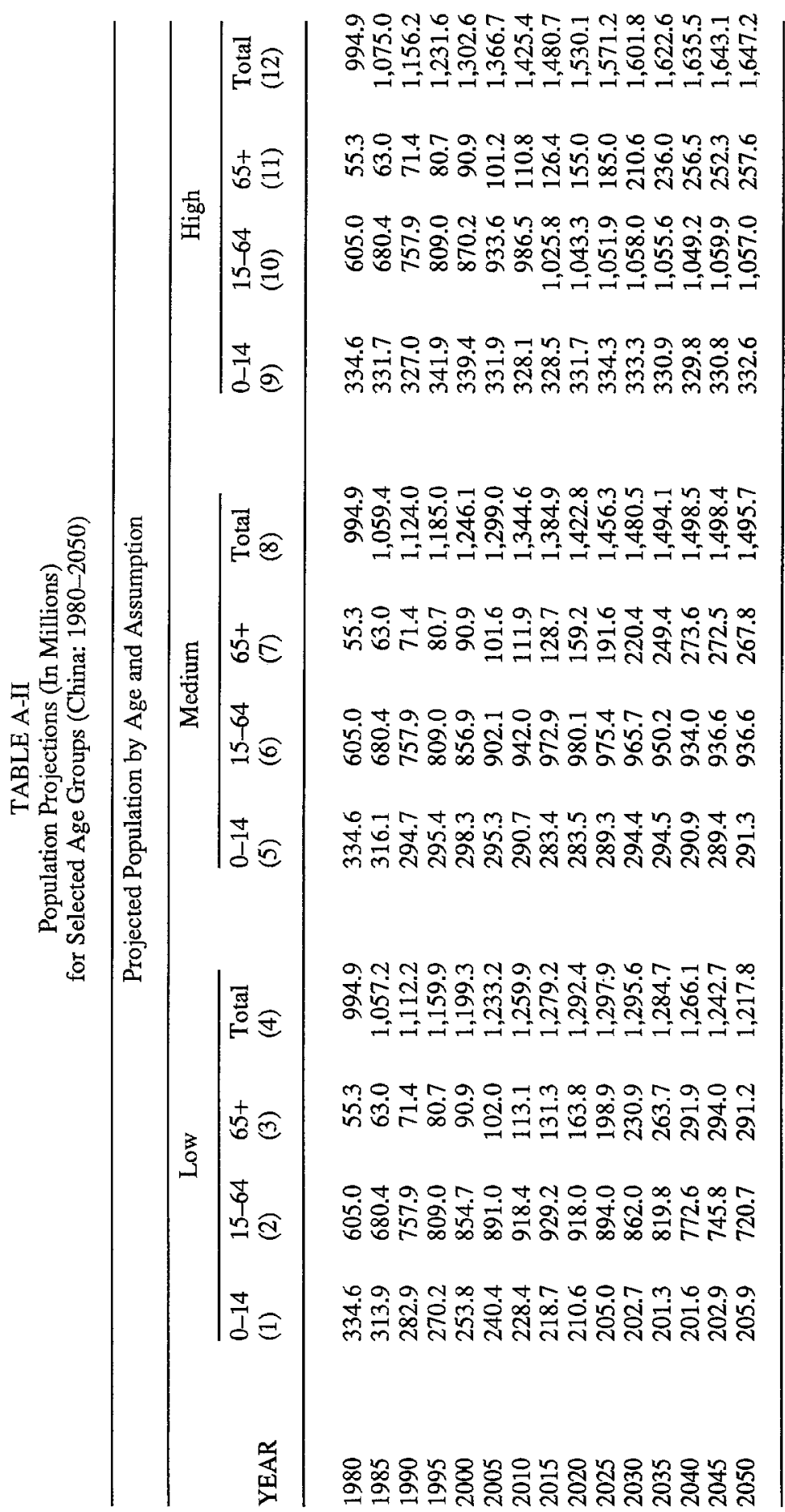

